\title{
Thomas-Fermi approximation for Bose-Einstein condensates in traps
}

\author{
P. Schuck ${ }^{1}$ and X. Viñas ${ }^{2}$ \\ ${ }^{1}$ Institut des Sciences Nucléaires, Université Joseph Fourier, CNRS-IN2P3, 53 Avenue des Martyrs, F-38026 Grenoble-Cédex, France \\ ${ }^{2}$ Departament d'Estructura i Constituents de la Matèria, Facultat de Física, Universitat de Barcelona, Diagonal 647, \\ E-08028 Barcelona, Spain
}

(Received 9 September 1999; revised manuscript received 24 November 1999; published 8 March 2000)

\begin{abstract}
Thomas-Fermi theory for Bose-Einstein condensates in inhomogeneous traps is revisited. The phase-space distribution function of the condensate in the Thomas-Fermi limit $(\hbar \rightarrow 0)$ is $f_{0}(\boldsymbol{R}, \boldsymbol{p}) \propto \delta\left(\mu-H_{c l}\right)$ where $H_{c l}$ is the classical counterpart of the self-consistent Gross-Pitaevskii Hamiltonian. Starting from this distribution function the Thomas-Fermi kinetic energy is calculated for any number of particles. Good agreement between the Gross-Pitaevskii and Thomas-Fermi kinetic energies is found even for low and intermediate particle numbers $N$. Application of this Thomas-Fermi theory to the attractive case and to the calculation of the frequencies of the monopole and quadrupole excitations in the sum rule approach yields conclusive results as well. The difference with the usual Thomas-Fermi approach to the Bose-Einstein condensates (large- $N$ limit) is discussed in detail.
\end{abstract}

PACS number(s): 03.75.Fi, 05.30.Jp

\section{INTRODUCTION}

The recent discovery of the Bose-Einstein condensation of magnetically trapped atoms has spurred a huge amount of theoretical investigations. Most of them are based on the Gross-Pitaevskii equation (GPE) [1], which is the mean-field equation for the condensate wave function (order parameter). The experimental conditions are such that the atomic gas is at very low density and therefore the mean-field approximation gives indeed excellent results [2-5]. Since the number $N$ of atoms involved is generally large, it is natural that also the Thomas-Fermi (TF) approximation is applied quite extensively. This has the advantage of yielding in most cases explicit analytical results of great physical transparency. The TF limit in the context of Bose-Einstein condensation in traps has been identified with the limit as the particle number $N$ goes to infinity. However, in the case of Fermi statistics, for which the TF approximation has been invented first [6], by no means the $\hbar \rightarrow 0$ limit (that is what the TF approximation really is) corresponds to the $N \rightarrow \infty$ limit. Rather the situation is such that the TF approach becomes eventually exact in the $N \rightarrow \infty$ limit but already for $N$ moderate, i.e., corresponding to the masses of midsized atoms, TF gives very reasonable estimates for various quantities such as ground-state energies, etc. It is the purpose of the present paper to show that also for the case of bosons the $N \rightarrow \infty$ limit is not equivalent to the $\hbar \rightarrow 0$ limit. Rather the correct $\hbar \rightarrow 0$ limit does not neglect the kinetic energy term in the GPE $(N \rightarrow \infty)$ and then the TF results are greatly improved for rather small mass numbers like, e.g., $N \approx 200$. We deliberately restrict ourselves here to the TF limit of the GPE at zero temperature. Finite temperature as well as more elaborated theories like the Bogoliubov approach may be the subject of future work.

The paper is organized as follows. In Sec. II the theoretical aspects of the TF approximation to the GPE are presented in detail. In Sec. III the numerical results obtained with the TF method are compared with the ones coming from the GPE. The last section is devoted to discussions and an outlook.

\section{GROSS-PITAEVSKII EQUATION. THOMAS-FERMI LIMIT}

As mentioned in the Introduction, the basic equation for Bose condensed atoms confined by magnetic traps is, in the low-density limit, given by the GPE for the wave function of the condensate

$$
\left(-\frac{\hbar^{2}}{2 m} \Delta+V_{e x}+g|\psi|^{2}\right) \psi=\mu \psi,
$$

where $V_{e x}$ is the external potential which for simplicity we have considered to be a spherical harmonic oscillator (for nonspherical geometry see remarks at the end of the paper). The coupling constant is given by $g=4 \pi \hbar^{2} a / m$ with $m$ the atomic mass and $a$ the scattering length. The chemical potential $\mu$ is identical with the lowest eigenvalue of the selfconsistent potential

$$
V=V_{e x}+g|\psi|^{2} .
$$

It is useful to note that Eq. (1) can also be rewritten as an equation for the density $\rho=|\psi|^{2}$

$$
\frac{\hbar^{2}}{2 m} \frac{1}{4}\left[\frac{(\nabla \rho)^{2}}{\rho^{2}}-2 \frac{\Delta \rho}{\rho}\right]+V_{e x}+g \rho-\mu=0 .
$$

In the large $N$ limit one can drop in Eq. (1) the kinetic energy or, equivalently, in Eq. (3) the gradients terms of the density. This leads to $\rho=\left(\mu-V_{e x}\right) / g$ what is known in the literature [3-5] as the TF solution of the GPE. However, for moderate particle numbers the kinetic energy is not negligible and there is no reason for dropping it in the proper TF limit ( $\hbar$ $\rightarrow \infty)$. Also in the attractive case the kinetic energy is of crucial importance to avoid collapse. 
As it is well known from the case of the Fermi systems [7], the TF-approximation is based on the assumption of a slowly varying potential so that its gradients can be neglected to lowest order. We now will show how this can be exploited in order to derive the zeroth order $\hbar$ expession for the solution of the GPE. To this end let us write Eq. (1) in the following way:

$$
(\mu-H) \hat{\rho}=0,
$$

where $H=p^{2} / 2 m+V_{e x}+g \rho$ is the GPE Hamiltonian and

$$
\hat{\rho}=|\psi\rangle\langle\psi|
$$

is the density matrix corresponding to the solution $\psi$ of Eq. (1). We note that lhs of Eq. (4) is an operator product of two single particle operators $\mu-H$ and $\hat{\rho}$. Taking the Wigner transform [7] of Eq. (4) we can use the fact the Wigner transform of a product of two single-particle operators $A$ and $B$ can be written as [7]

$$
(A B)_{\text {Wigner }}=A_{\text {Wigner }} \exp (i \hbar \kappa / 2) B_{\text {Wigner }},
$$

where

$$
\overleftrightarrow{\Lambda}=\grave{\nabla}_{r} \cdot \vec{\nabla}_{p}-\overleftarrow{\nabla}_{p} \cdot \vec{\nabla}_{r}
$$

is the Poisson bracket operator and the upper arrows indicate in which direction the differentation should act.

Explicitly to order $\hbar^{2}$ expression (6) is for example given in Ref. [8]. Since we can symmetrize Eq. (4) we can write with Eq. (6)

$$
\left[\mu-H_{c l}(\boldsymbol{R}, \boldsymbol{p})\right] \cos \frac{\hbar}{2} \overleftrightarrow{\Lambda} f(\boldsymbol{R}, \boldsymbol{p})=0,
$$

where $H_{c l}$ is the classical counterpart of the Hamiltonian in the GPE and $f(\boldsymbol{R}, \boldsymbol{p})$ is the Wigner transform of the density matrix $\hat{\rho}$ of Eq. (5). To lowest order in $\hbar$ we obtain from Eq. (8)

$$
\left(\mu-H_{c l}\right) f_{0}(\boldsymbol{R}, \boldsymbol{p})=0
$$

what is a $c$-number equation. With $x \delta(x)=0$ we find for the solution of Eq. (9)

$$
f_{0}(\boldsymbol{R}, \boldsymbol{p})=N c \delta\left(\mu-H_{c l}\right)
$$

where

$$
\frac{1}{c}=g(\mu)=\int \frac{d \boldsymbol{R} d \boldsymbol{p}}{(2 \pi \hbar)^{3}} \delta\left(\mu-H_{c l}\right)
$$

is the level density and thus $\rho_{0}$ is correctly normalized to the particle number $N$. We would like to point out that Eq. (10) is not a new result but actually textbook knowledge (see for example the monograph by Gutzwiller [9]). Let us mention again the fact that Eq. (10) has been derived under the sole assumption that all the gradients of the potential can be neglected, which is the usual hypothesis of TF theory. Clearly, contrary to what has become known as "TF approximation" for trapped atomic bosons [3-5], expression (10) does not neglect the kinetic energy. Equation (10) also is consistent with the usual TF theory for fermions where not only the lowest level is filled but all levels below the Fermi energy. One therefore simply has to sum all $\delta$ functions (10) over the energies of the levels. Converting the discrete sum into an integral and introducing an extra level density, one obtains

$$
\Theta\left(\mu-H_{c l}\right)=2 \int_{0}^{\mu} d E \delta\left(E-H_{c l}\right),
$$

where the factor 2 stands for the spin-degeneracy. The lhs of Eq. (12) is recognized as the standard TF expression for the distribution function of a degenerate Fermi gas [7].

The main point of this paper is to show that Eq. (10) leads to a more complex expression for the density $\rho(\boldsymbol{R})$ $=\int d \boldsymbol{p} f_{0}(\boldsymbol{R}, \boldsymbol{p}) /(2 \pi \hbar)^{3}$, which however, reduces in the limit of large numbers $N$ to the usual form $\rho=\left(\mu-V_{e x}\right) / g$. This will be the subject of the following paragraphs where it will also be demostrated that Eq. (10) gives very reasonable results for observables already for modest number of particles, strongly improving over the large $N$ limit.

Before coming to these points we would like to contrast our approach to the one published in Ref. [8] by Timmermans et al. The essential point in Ref. [8] is the semiclassical treatment of scattering processes out of the condensate in the Bogoliubov approach. Though this is certainly a worthwhile generalization of the Gross-Pitaevskii approach to be considered semiclassically, a sensible comparison with our theory can only be performed once the Bogoliubov terms in Ref. [8] have been switched off. In detail this means that in a general Bogoliubov transformation to quasiparticles, i.e., $\alpha_{k}^{\dagger}=u_{k} a_{k}^{\dagger}$ $-v_{k} a_{k}+C$ we have to choose $v_{k}=0$ and $u_{k}=1$, since only $v_{k}=0$ corresponds to the GPE and any $v_{k} \neq 0$ means that a certain depopulation of the condensate due to correlation is present. A careful inspection of the formalism presented in Ref. [8] shows that on the level of GPE the approach of those authors reduces to the ususal, i.e., to the large $N$ limit. We therefore see that in the context of condensation of atomic atoms in magnetic traps expression (10) leads to results turning the TF approach to GPE into a quite reliable one even for very moderate numbers of particles. This we will demostrate in Sec. III.

\section{A. Self-consistent solution. Repulsive case $(g>0)$}

Let us first consider the solution of the self-consistent problem at the TF level defined by Eq. (10) for the repulsive case i.e., $g>0$. From Eq. (10) we obtain for the density:

$$
\rho(\boldsymbol{R})=\int \frac{d \boldsymbol{p}}{(2 \pi \hbar)^{3}} f_{0}(\boldsymbol{R}, \boldsymbol{p})=\frac{N c m}{2 \pi^{2} \hbar^{3}} p_{0}(\boldsymbol{R}),
$$

where the local momentum is given by

$$
p_{0}(\boldsymbol{R})=\sqrt{2 m\left(\mu-V_{e x}-g \rho\right)} .
$$

The self-consistency between Eqs. (13) and (14) is easy to solve analytically and we obtain 


$$
\rho=-\frac{K g N^{2}}{2}+\sqrt{\left(\frac{K g N^{2}}{2}\right)^{2}+K N^{2}\left(\mu-V_{e x}\right)},
$$

where

$$
K=\frac{2 m}{\hbar^{2}}\left(\frac{c m}{2 \pi^{2} \hbar^{2}}\right)^{2}
$$

It is to be noted that $\rho(\boldsymbol{R})$ is defined only within the classical region limited by $\mu-V_{e x}(\boldsymbol{R})=0$. It is straightforward to expand $\rho$ in the repulsive case $g>0$ for large values of $N$

$$
\rho \approx \frac{1}{g}\left(\mu-V_{e x}\right)-\frac{1}{K g^{3} N^{2}}\left(\mu-V_{e x}\right)^{2}+\cdots .
$$

It is satisfying to see that to leading order one recovers the result corresponding to the total neglect of kinetic energy in Eq. (1) (see Introduction).

The normalization is directly determined from Eq. (11)

$$
\frac{1}{c}=\frac{m}{2 \pi^{2} \hbar^{3}} \int d \boldsymbol{R} p_{0}(\boldsymbol{R})
$$

or equivalently

$$
N=\int d \boldsymbol{R} \rho(\boldsymbol{R})
$$

Explicitly one obtains from this equation

$$
\begin{aligned}
1= & 4 \pi\left(\frac{2}{m \omega^{2}}\right)^{3 / 2}\left\{-\frac{5 K g N}{48} \mu^{3 / 2}-\frac{K^{2} g^{3} N^{3}}{64} \mu^{1 / 2}\right. \\
& \left.+\frac{\sqrt{K}}{8}\left(\frac{K g^{2} N^{2}}{4}+\mu\right)^{2} \arcsin \sqrt{\frac{\mu}{\frac{K g^{2} N^{2}}{4}+\mu}}\right\} .
\end{aligned}
$$

Using in Eq. (19) the asymptotic expansion (17) yields

$$
1=4 \pi\left(\frac{2}{m \omega^{2}}\right)^{3 / 2} \frac{2}{15} \frac{\mu^{5 / 2}}{g N}\left(1-\frac{4}{7} \frac{\mu}{K g^{2} N^{2}}\right),
$$

which also can directly be derived in expanding Eq. (20).

From Eq. (20) or (21) we can determine the normalization constant $c$ as a function of $\mu$. From Eq. (21) we see that to lowest order in $1 / N$ the normalization constant $c$ drops out and thus in this limit the chemical potential is, as usual, determined by the particle number condition. However, as we will see, via the quantization condition $\mu$ depends on $c$ and thus we can consider Eq. (20) as determining the normalization in any case.

\section{B. Chemical potential and quantization}

The semiclassical density matrix (10) corresponds to a single wave function. In such a case we have two independent constants to be determined: the normalization " $c$ " and the chemical potential $\mu$, i.e., the lowest eigenvalue of the GPE. Only in the case $N \rightarrow \infty$ does the chemical potential coincide with the bottom of the (self-consistently determined) potential well and no extra determination of $\mu$ is necessary. For finite number of particles a requantization of the lowest order semiclassical theory, i.e., TF approximation in this case, is necessary in order to determine (semiclassically) the finite gap separating the lowest eigenvalue of the GPE from the bottom of the potential. This procedure is similar to the Wentzel-Kramers-Brillouin (WKB) requantization. The need for a requantization of the TF theory for individual states has been recognized in Ref. [10] and in fact our procedure of requantization clearly follows what is proposed there. One may think that the equation for the chemical potential $\mu=d E / d N$ yields the right quantization but in fact is equivalent to the particle number condition (19) and therefore of no help. The standard semiclassical quantization procedure is given by the WKB method. However, in order to have a more explicit formula, we here also apply a slightly simpler method, applicable to the lowest state in a singleparticle potential [11]. To this end we calculate the smooth accumulated level density (number of states) in TF approximation

$$
N^{T F}(E)=\int \frac{d \boldsymbol{R} d \boldsymbol{p}}{(2 \pi \hbar)^{3}} \Theta\left(E-H_{c}\right)
$$

For a spherical harmonic oscillator $(\mathrm{HO})$ this gives

$$
N_{H . O .}^{T F}=\frac{1}{6}\left(\frac{E}{\hbar \omega}\right)^{3}
$$

Taking for $E$ in Eq. (23) the $\mathrm{HO}$ eigenvalues

$$
E \rightarrow E_{K}=\left(K+\frac{3}{2}\right) \hbar \omega
$$

with $K=2 n+l$ and inserting Eqs. (23) and (24) in the lefthand side of Eq. (22) yields a semiclassical quantization rule, which becomes exact in the three-dimensional (3D) spherical $\mathrm{HO}$ case. It represents an approximate quantization relation for an arbitrary potential where the quantized energies very well reproduce the centroid of major shells. This has been tested numerically on a potential of Woods-Saxon type of nuclear dimensions [11]. It is evident that in the 1D case the same procedure leads to the exact WKB quantization rule. For the $3 \mathrm{D}$ case this modified quantization prescription is slightly less accurate than WKB for the lowest eigenvalue but has the advantage to be easier and to be readily applicable also to the deformed case [11]. In the present problem, the eigenvalue $\mu$ is then determined by 


$$
\begin{gathered}
\frac{27}{8}=\frac{1}{\pi^{2} \hbar^{3}} \int d \boldsymbol{R} p_{0}^{3}(\boldsymbol{R}) \\
=\frac{32}{\pi \hbar^{3}}\left\{-\frac{27}{80}\left(\frac{K g^{2} N^{2}}{4}\right)^{1 / 2} \mu^{5 / 2}-\frac{19}{24}\left(\frac{K g^{2} N^{2}}{4}\right)^{3 / 2} \mu^{3 / 2}\right. \\
-\frac{7}{16}\left(\frac{K g^{2} N^{2}}{4}\right)^{5 / 2} \mu^{1 / 2} \\
+\frac{3}{8} \frac{K g^{2} N^{2}}{4}\left(\frac{K g^{2} N^{2}}{4}+\mu\right)^{2} \arcsin \sqrt{\frac{\mu g^{2} N^{2}}{4}+\mu} \\
\left.+\frac{1}{16}\left(\frac{K g^{2} N^{2}}{4}+\mu\right)^{3} \arcsin \sqrt{\frac{\mu}{\frac{K g^{2} N^{2}}{4}+\mu}}\right\},
\end{gathered}
$$

where we have used $p_{0}(\boldsymbol{R})$ from Eq. (14) with Eq. (15). To second order we obtain from Eq. (25)

$$
\begin{aligned}
\frac{27}{8}= & \frac{4}{\pi \hbar^{3}}\left(\sqrt{\frac{2 m}{K}} \frac{1}{g N}\right)^{3}\left(\frac{2}{m \omega^{2}}\right)^{3 / 2} \\
& \times \mu^{9 / 2} \frac{16}{315}\left(1-\frac{48}{11} \frac{\mu}{K g^{2} N^{2}}\right) .
\end{aligned}
$$

To leading order in the large $N$ limit we obtain from Eqs. (20) and (21) and Eqs. (25) and (26)

$$
\begin{gathered}
\mu_{0}=\left(\frac{15}{8 \pi}\right)^{2 / 5}\left(\frac{m \omega^{2}}{2}\right)^{3 / 5}(g N)^{2 / 5} \\
K_{0}=\left(\frac{4096}{8505 \pi \hbar^{3} \omega^{3}}\right)^{2 / 3} \frac{1}{(g N)^{2}} \mu_{0}^{3} .
\end{gathered}
$$

This completes the solution in the large $N$ limit.

For later comparison let us also give the standard WKB quantization rule [12], which we want to evaluate to leading order

$$
\frac{\pi}{2}=\sqrt{\frac{2 m}{\hbar^{2}}} \int_{r_{1}}^{r_{2}} d r\left[\mu-V(r)-\frac{\hbar^{2}}{2 m} \frac{1 / 4}{r^{2}}\right]
$$

with [see Eq. (17)]

$$
\mu-V(r)=\frac{1}{K g^{2} N^{2}}\left[\mu-V_{e x}(r)\right]^{2} .
$$

The classical turning points $r_{1}$ and $r_{2}$ are determined from the solution of the cubic equation

$$
\frac{1}{\sqrt{K g^{2} N^{2}}}\left(\mu-V_{e x}\right)=-\frac{1}{2} \sqrt{\frac{\hbar^{2}}{2 m}} \frac{1}{r} .
$$

With Eqs. (29) and (30), Eq. (28) can be solved for $\mu$.

\section{Kinetic energy}

One of the main difficulties with the standard $N \rightarrow \infty$ TF limit treated in the literature (see Introduction) consists in the inability to calculate the kinetic energy [3]. In our approach this does not cause any particular problem and one directly obtains

$$
E_{k i n}=\int \frac{d \boldsymbol{R} d \boldsymbol{p}}{(2 \pi \hbar)^{3}} \frac{p^{2}}{2 m} f_{0}(\boldsymbol{R}, \boldsymbol{p})=\frac{N c}{4 \pi^{2} \hbar^{3}} \int d \boldsymbol{R} p_{0}^{3}(\boldsymbol{R}) .
$$

For example to lowest order we obtain from Eqs. (14) and (17)

$$
p_{0}=\frac{1}{\sqrt{K_{0}} g N}\left(\mu_{0}-V_{e x}\right) \Theta\left(\mu_{0}-V_{e x}\right)
$$

what yields for the kinetic energy per particle, using Eq. (27)

$$
\frac{E_{\text {kin } 0}^{(0)}}{N}=\frac{27}{32} \frac{2 \pi^{2} \hbar^{3}}{m} \sqrt{\frac{K_{0}}{2 m}}=\frac{27}{32} c_{0} .
$$

This simple result must be contrasted with the usual statment that in the $N \rightarrow \infty$ TF limit the kinetic energy cannot be evaluated, since it diverges [3].

Indeed one can write the kinetic energy as

$$
E_{k i n}=\frac{\hbar^{2}}{2 m} \int d \boldsymbol{R}|\nabla \psi|^{2}
$$

In the $N \rightarrow \infty$ limit we have

$$
\psi_{N \rightarrow \infty}=\sqrt{\frac{1}{g}\left(\mu_{0}-V_{e x}\right)}
$$

and one can readily verify that with Eq. (35) $E_{k i n}$ of Eq. (34) diverges logarithmically. This result obviously is in contradiction with Eq. (33) and we shortly want to elucidate the underlying reason. To this end, we first rewrite Eq. (34) in a different but obviously equivalent way:

$$
E_{k i n}=\frac{\hbar^{2}}{2 m} \int d \boldsymbol{R}|\nabla \psi|^{2}=\int \frac{d \boldsymbol{R} d \boldsymbol{p}}{(2 \pi \hbar)^{3}} \frac{p^{2}}{2 m} \widetilde{f}_{0}(\boldsymbol{R}, \boldsymbol{p})
$$

with $\widetilde{f}_{0}$ given by the Wigner transform of the density matrix corresponding to Eq. (35):

$$
\tilde{f}_{0}(\boldsymbol{R}, \boldsymbol{p})=\int d \boldsymbol{s} e^{-i p s / \hbar} \psi_{N \rightarrow \infty}\left(\boldsymbol{R}+\frac{\boldsymbol{s}}{2}\right) \psi_{N \rightarrow \infty}\left(\boldsymbol{R}-\frac{\boldsymbol{s}}{2}\right) .
$$

Since $\widetilde{f}_{0} \neq f_{0}$ we argue that Eq. (37) is not the correct $\hbar$ $\rightarrow 0$ limit of the Wigner phase space function because it is not the solution of the $\hbar \rightarrow 0$ limit of the Schrödinger Eq. (1) given by Eq. (9). Only (10) is the correct solution of this equation, which yields for large $N$ 


$$
f_{0_{N \rightarrow \infty}}=N c \delta\left[\frac{1}{K_{0} g^{2} N^{2}}\left(\mu_{0}-V_{e x}\right)^{2}-\frac{p^{2}}{2 m}\right] .
$$

One checks that Eq. (38) upon projection onto r-space gives the correct lowest order expression for the density [see Eq. (17)]. Therefore both Wigner functions (37) and (38) yield the same leading order density. However, in spite of being a very suggestive nonlocal generalization of the lowest order local density expression, Eq. (37) has to be rejected on the above given grounds and the divergency of Eq. (34) is an artifact. On the contrary the lowest order contribution to the kinetic energy is given by Eq. (33). Via Eqs. (21) and (26) it is straightforward to calculate the next to leading order correction to Eq. (33). It should, however, be remembered that $1 / N$ corrections do not go in parallel with powers in $\hbar$ and that $1 / N$ corrections also can come from $\hbar^{2}$ corrections to Eq. (10), which involve second-order gradients of the potential. In any case, the Wigner-Kirkwood expansion of the density matrix is an asymptotic expansion, which in no way can recover the nonanalytic behavior in $\hbar$ of the quantal solution. In the present problem, the nonanalyticity in $\hbar$ of the quantal solution entails a nonanalytic behavior in $1 / N$ [see Eq. (16) of Ref. [3]] and therefore a WK-expansion can never recover the quantal behavior in $1 / N$. It is well known that an asymptotic expansion has to be stopped at a point where the difference to the exact solution is minimal. Afterwards the expansion starts to diverge again. In this paper, we do not intend to develop a systematic expansion simultaneously in $\hbar$ and $1 / N$. We rather want to give a complete solution to lowest order in $\hbar$, i.e., on the TF level.

\section{The attractive case $(g<0)$}

Recently, Bose-Einstein condensation has also been observed for the case of negative scattering length $\left({ }^{11} \mathrm{Li}\right.$ atoms) [13]. For $g<0$ the Gross-Pitaevskii approach leads to metastability for particle numbers $N \leqslant 1400$ [2]. For large particle numbers the system collapses. For the attractive case ( $a$ $<0)$ the correct treatment of the kinetic energy is crucial in the TF limit, since otherwise no stability can be achieved. Formally the TF solution for the density is the same as in Eq. (15) with, however, the sign of the first member reversed

$$
\rho=\frac{K|g| N^{2}}{2}+\sqrt{\left(\frac{K g N^{2}}{2}\right)^{2}+K N^{2}\left(\mu-V_{e x}\right)} .
$$

Contrary to the repulsive case no large $N$ expansion is possible here. Therefore, the TF solution has to be considered in full. In the next section, comparison with quantal results will be given.

Let us summarize our findings of this section. It has been pointed out that what in the literature is usually called the Thomas-Fermi approximation of the GPE, i.e., its $N \rightarrow \infty$ limit, is not equivalent to the limit $\hbar \rightarrow 0$ of the GPE. In an analogy with the case of fermions, where the TF approximation corresponds to the limit $\hbar \rightarrow 0$ of the quantal solution, we have also elaborated the "true" TF approximation in taking properly the limit $\hbar \rightarrow 0$. Taking in addition the large
$N$ limit makes this coincide with the ususal $N \rightarrow \infty$ limit of the GPE. However, the limit $\hbar \rightarrow 0$ of the GPE has the advantage that it gives very good estimates of various quantities (including kinetic energy) already for rather small number of particles $(N=200-300)$ and it can be applied to the attractive $(g<0)$ case, as will be demostrated in the next section. Of course, as a rue, the $(\hbar=0) \mathrm{TF}$ approximation works better for large number of particles.

\section{NUMERICAL RESULTS}

In this section, we proceed to a detailed numerical comparison of the semiclassical approximations with the exact quantum mechanical results. Along this section, energies and lengths are given in harmonic oscillator units: $\hbar \omega$ and $a_{H O}$ $=\sqrt{\hbar / 2 m \omega}$, respectively. First, in Table I we present the chemical potential $(\mu)$ and the kinetic $\left(e_{k i n}\right)$, harmonic oscillator $\left(e_{H O}\right)$ and the self-interaction $\left(e_{\rho}\right)$ energies per particle calculated quantally and in the full Thomas-Fermi approximation (15), (20) and (25) as a function of the number of atoms enclosed in the trap. We have considered Cs atoms (as was done in Ref. [14]), the frequency of the harmonic oscillator has been chosen to be $\omega=20 \pi s^{-1}$ and the scattering length to be $a=3.2 \times 10^{-9} \mathrm{~m}$.

In Table II, we present the results for the chemical potential and the kinetic energy per particle number beyond 20000 . In addition to the quantum mechanical and the full Thomas-Fermi results, we also include the results for the large $N$ limit (33) and those obtained using the WKB quantization rule (in the large $N$ limit). Notice that in the large $N$ limit, the WKB chemical potential coincides with the TF one and the kinetic energy is given also by Eq. (33) but with $c_{0}$ replaced by the one calculated from $K$ via Eq. (28). Though globally the semiclassical $(\hbar=0)$ TF results reported in Tables I and II are in quite satisfactory agreement with the corresponding quantal values, one nevertheless remarks upon some unexpected features. For instance the kinetic energy in the $\mathrm{TF}$ approximation is larger than the exact values for small numbers of particles whereas it undershoots the quantum values quite considerably in the large $N$ limit. To clarify this point we will discuss with some detail the results obtained for a very large number of particles which are collected in Table II.

The numbers presented in this table indicate that the values of the chemical potential $\mu$ obtained quantally and using the $(\hbar=0)$ TF approach (columns 1 and 2 of Table II) only reach its asymptotic TF limit (column 3 ) for a very large number of particles $\left(N \simeq 10^{8}\right)$. Although the chemical potential reported in columnn 3 is the large $N$ limit (27) of our $(\hbar=0) \mathrm{TF}$ theory, it coincides with the corresponding value obtained in the standard $(N \rightarrow \infty)$ TF approach. For a relatively large number of particles the value of $\mu$ obtained with the $(\hbar=0)$ TF approach lies halfway in between the limiting $(N \rightarrow \infty)$ TF and the quantal results. So even for a very large number of particles the $(\hbar=0)$ TF approximation shows a clear superiority over its $N \rightarrow \infty$ limit.

Concerning the kinetic energy we first want to point out that with the standard $N \rightarrow \infty$ TF theory, as it is well known [3], it is impossible to calculate the kinetic energy. The rea- 
TABLE I. Chemical potential $(\mu)$, kinetic energy $\left(e_{k i n}\right)$, harmonic oscillator energy $\left(e_{H O}\right)$, and selfinteraction energy $\left(e_{\rho}\right)$ per particle in harmonic oscillator units $(\hbar \omega)$ calculated quantally $(\mathrm{QM})$ and in the Thomas-Fermi approximation (TF) for several numbers of atoms in the traps. The frequency of the harmonic oscillator is $\omega=20 \pi \mathrm{s}^{-1}$ and the scattering length is $\mathrm{a}=3.2 \times 10^{-9} \mathrm{~m}$.

\begin{tabular}{rcccccccc}
\hline \hline \multicolumn{1}{c}{$N$} & $\mu(\mathrm{QM})$ & $\mu(\mathrm{TF})$ & $e_{k i n}(\mathrm{QM})$ & $e_{k i n}(\mathrm{TF})$ & $e_{H O}(\mathrm{QM})$ & $e_{H O}(\mathrm{TF})$ & $e_{\rho}(\mathrm{QM})$ & $e_{\rho}(\mathrm{TF})$ \\
\hline 200 & 1.688 & 1.642 & 0.696 & 0.700 & 0.811 & 0.804 & 0.080 & 0.069 \\
400 & 1.806 & 1.766 & 0.654 & 0.661 & 0.865 & 0.851 & 0.144 & 0.127 \\
600 & 1.927 & 1.877 & 0.622 & 0.630 & 0.912 & 0.894 & 0.196 & 0.177 \\
800 & 2.036 & 1.978 & 0.597 & 0.603 & 0.955 & 0.934 & 0.242 & 0.220 \\
1000 & 2.134 & 2.071 & 0.575 & 0.581 & 0.944 & 0.970 & 0.282 & 0.260 \\
1200 & 2.225 & 2.157 & 0.557 & 0.561 & 1.031 & 1.005 & 0.319 & 0.296 \\
1400 & 2.310 & 2.238 & 0.541 & 0.544 & 1.065 & 1.037 & 0.352 & 0.329 \\
1600 & 2.389 & 2.315 & 0.528 & 0.528 & 1.065 & 1.068 & 0.382 & 0.359 \\
1800 & 2.464 & 2.388 & 0.515 & 0.514 & 1.097 & 1.097 & 0.411 & 0.388 \\
2000 & 2.535 & 2.457 & 0.503 & 0.502 & 1.158 & 1.125 & 0.437 & 0.415 \\
4000 & 3.112 & 3.025 & 0.431 & 0.417 & 1.395 & 1.356 & 0.643 & 0.626 \\
6000 & 3.550 & 3.461 & 0.390 & 0.369 & 1.577 & 1.536 & 0.792 & 0.778 \\
8000 & 3.914 & 3.825 & 0.363 & 0.336 & 1.729 & 1.687 & 0.911 & 0.901 \\
10000 & 4.231 & 4.142 & 0.343 & 0.312 & 1.862 & 1.820 & 1.013 & 1.005 \\
12000 & 4.513 & 4.426 & 0.327 & 0.293 & 1.981 & 1.939 & 1.103 & 1.097 \\
14000 & 4.770 & 4.684 & 0.314 & 0.277 & 2.089 & 2.047 & 1.184 & 1.180 \\
16000 & 5.007 & 4.921 & 0.303 & 0.265 & 2.189 & 2.147 & 1.258 & 1.255 \\
18000 & 5.228 & 5.143 & 0.294 & 0.254 & 2.282 & 2.240 & 1.326 & 1.324 \\
20000 & 5.435 & 5.350 & 0.285 & 0.244 & 2.369 & 2.328 & 1.390 & 1.389 \\
\hline \hline
\end{tabular}

son for this shortcoming has been discussed in Sec. II C. Since the $(\hbar=0)$ TF theory does not neglect the kinetic energy it can be perfectly calculated (column 5 of Table II). One can also consider the leading term for large $N$ of the $(\hbar=0) \mathrm{TF}$ expression for the kinetic energy. This is given by Eq. (33) and the corresponding values are displayed in column 6 of Table II. We see that the $(\hbar=0)$ TF kinetic energy reaches this limiting value for a rather moderate number of particles $\left(N \simeq 10^{5}\right)$. We also realize that the $(\hbar=0)$ TF kinetic energy, that is derived with our simplified quantization rule (25) and (26), coincides with the value obtained using the WKB quantization rule (column 7 of Table II) for a very large number of particles $\left(N \simeq 10^{7}-10^{8}\right)$. From Table II it is also seen that even for a very large number of particles

TABLE II. Chemical potential $(\mu)$ and kinetic energy $\left(e_{k i n}\right)$ per particle for large number of atoms in the trap $(N)$. The chemical potential is calculated quantally $(\mathrm{QM})$, with the full Thomas-Fermi approach $(\mathrm{TF})$ and with the asymptotic formula for large $N\left(\mathrm{TF}_{N \rightarrow \infty}\right)$. The kinetic energy is obtained quantally, with the exact TF approximation, with the asymptotic TF for large $N$ and using the WKB quantization in the limit of large number of atoms. The frequency of the harmonic oscillator and the scattering lenght are the same as in Table I.

\begin{tabular}{rrrccccc}
\hline \hline \multicolumn{1}{c}{$N$} & $\mu(\mathrm{QM})$ & $\mu(\mathrm{TF})$ & $\mu\left(\mathrm{TF}_{N \rightarrow \infty}\right)$ & $e_{k i n}(\mathrm{QM})$ & $e_{k i n}(\mathrm{TF})$ & $e_{k i n}\left(\mathrm{TF}_{N \rightarrow \infty}\right)$ & $e_{k i n}\left(\mathrm{WKB}_{N \rightarrow \infty}\right)$ \\
\hline 20000 & 5.435 & 5.350 & 5.196 & 0.285 & 0.244 & 0.256 & 0.238 \\
30000 & 6.322 & 6.242 & 6.111 & 0.255 & 0.210 & 0.218 & 0.202 \\
40000 & 7.051 & 6.973 & 6.856 & 0.236 & 0.187 & 0.194 & 0.180 \\
50000 & 7.677 & 7.603 & 7.496 & 0.222 & 0.173 & 0.177 & 0.165 \\
100000 & 10.231 & 9.972 & 9.891 & 0.182 & 0.133 & 0.134 & 0.125 \\
150000 & 11.763 & 11.701 & 11.633 & 0.162 & 0.113 & 0.114 & 0.106 \\
200000 & 13.170 & 13.112 & 13.051 & 0.149 & 0.101 & 0.102 & 0.095 \\
250000 & 14.381 & 14.326 & 14.270 & 0.140 & 0.093 & 0.093 & 0.087 \\
500000 & 18.919 & 18.872 & 18.829 & 0.114 & 0.070 & 0.070 & 0.066 \\
1000000 & 24.917 & 24.878 & 24.845 & 0.092 & 0.053 & 0.053 & 0.050 \\
5000000 & 47.340 & 47.340 & 47.297 & 0.056 & 0.028 & 0.028 & 0.026 \\
10000000 & 62.443 & 62.422 & 62.409 & 0.045 & 0.021 & 0.021 & 0.020 \\
25000000 & 90.056 & 90.046 & 90.037 & 0.033 & 0.015 & 0.015 & 0.014 \\
\hline \hline
\end{tabular}




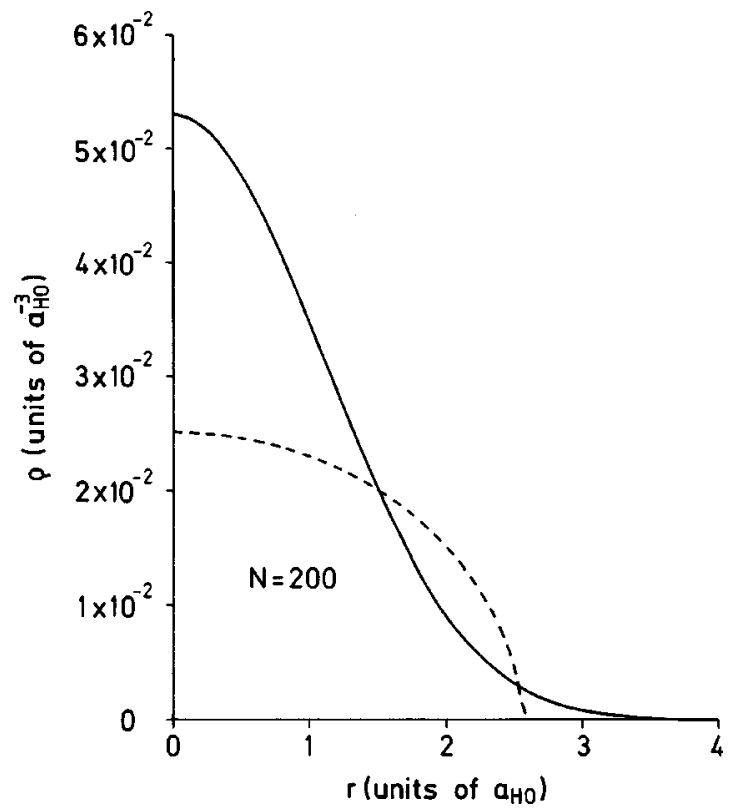

FIG. 1. Density (normalized to unity) of 200 atoms in a spherical trap (in $a_{H O}^{-3}$ units) as a function of the distance (in $a_{H O}$ units) in the repulsive case calculated from the solution of the GPE (solid line) and using the TF approach described in the text (dashed line).

$\left(N \simeq 10^{8}\right)$ the kinetic energy $(\hbar=0)$ TF values are still different (smaller) than the corresponding quantal results. This is due to the fact that for very large $N$ the quantal kinetic energy is dominated by the quantal corrections. As was shown by Dalfovo, Pitaevskii, and Stringari [3], these corrections are nonanalytical (logarithmic) in $\hbar$. Consequently, they cannot be accounted for by a pure TF approach and a partial resummation of all orders in $\hbar$ would be necessary to reproduce the quantal kinetic energy. Of course, when finally $N \rightarrow \infty$ these quantal corrections drop to zero and one recovers the result of the standard TF theory where the kinetic energy vanishes.

Next, let us compare in Figs. 1 and 2 the densities (normalized to unity) in TF-approximation and calculated exactly for small (200) and large (200 000) particle numbers. As expected, the TF densities almost agree with the quantal ones for very large particle numbers. In view of the still quite reasonable expectation values shown in Table I for $N=200$, the strong deviation of the TF density from the quantal result is somewhat a surprise. However, one always should remember that the TF solution for the densities is to be understood as a distribution [see Eq. (10)], which for expectation values of "slowly varying" operators can still yield very reasonable values in spite of the fact that the detailed shape may only be a caricature of the exact one.

In Figs. 3 and 4 we show the self-consistent potentials $V=V_{e x}+g \rho$ corresponding to the densities of Figs. 1 and 2. Not astonishingly, $V$ deviates from the harmonic oscillator only slightly for $N=200$, both quantally and semiclassically. On the contrary for $N=200000$, the potential $V$ deviates strongly from $V_{e x}$ being practically a constant equal to $\mu$ up to the classical turning point from where the harmonic oscillator takes over quite abruptly. Again, both the quantal and

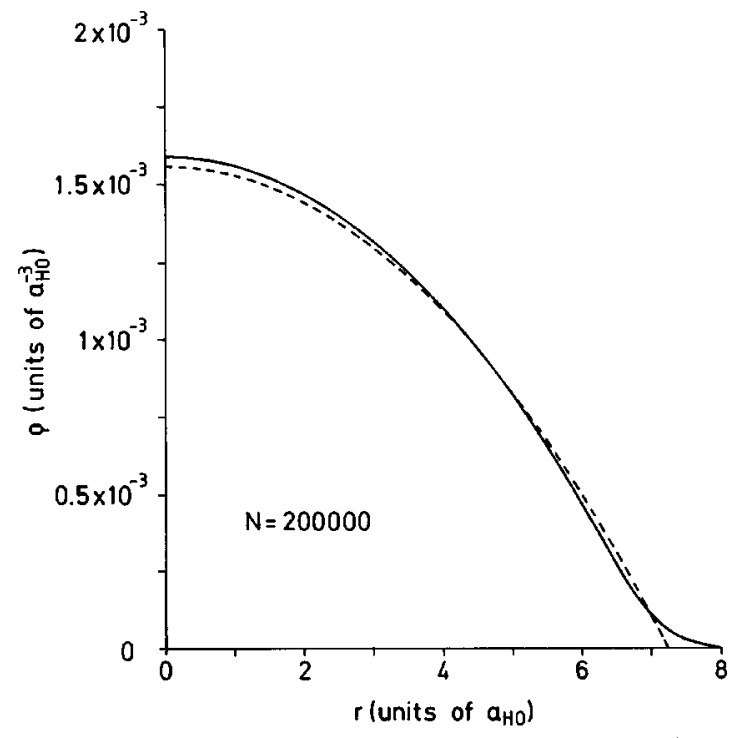

FIG. 2. The same as Fig. 1 but with 200000 atoms in the trap.

TF-solution are in close agreement. Figure 4 also teaches us why the TF approximation (10) to the quantal distribution function is very good for large $N$. The distribution function corresponds to a wave function with very large energy $\mu$. In phase space it therefore is very much concentrated around the surface of the hypersphere with radius $\mu$.

Let us now present the attractive case for the same atoms and external potential with, however, the scattering length $a=-1.0 \times 10^{-9} \mathrm{~m}$. In Table III we again show chemical potential and kinetic, harmonic oscillator, and self-interaction energies per particle as a function of the particle number in $\mathrm{TF}$ and quantal calculation. For small particle numbers $(N$ $\leqslant 1000$ ) the agreement of TF with the quantal case is of

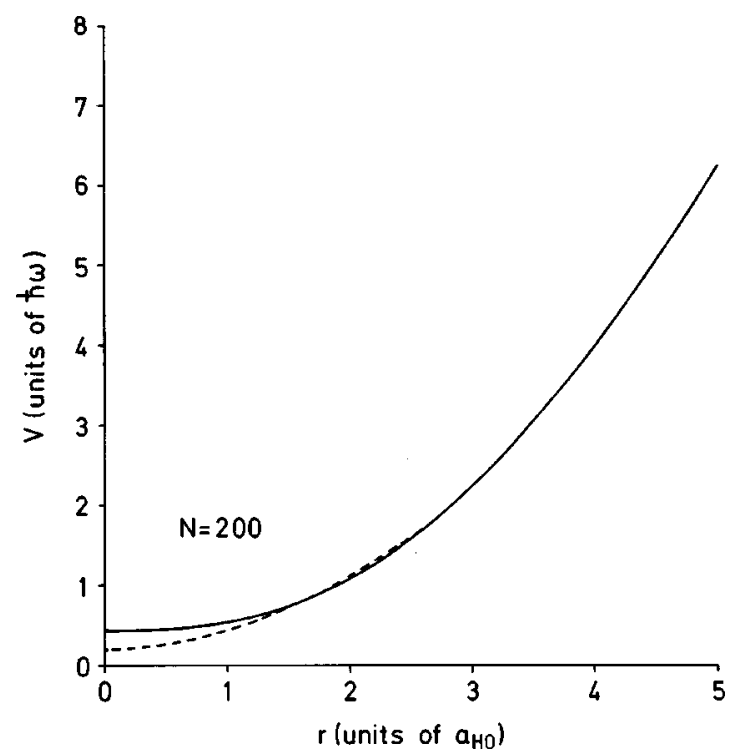

FIG. 3. Self-consistent potential (in $\hbar \omega$ units) corresponding to a spherical trap containing 200 atoms as a function of the distance ( $a_{H O}$ units) in the repulsive case calculated from the solution of the GPE (solid line) and using the TF approach described in the text (dashed line). 


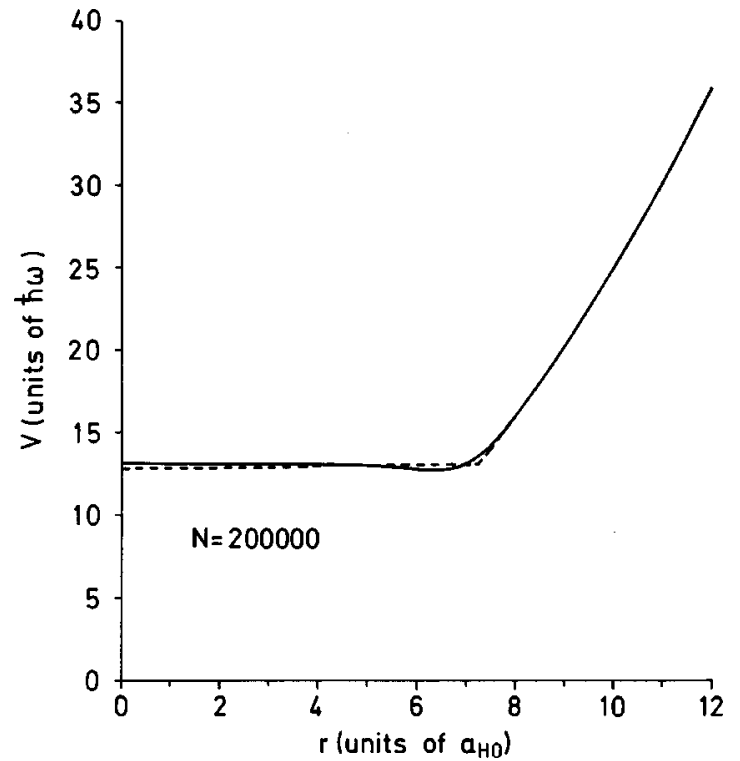

FIG. 4. The same as Fig. 3 but with 200000 atoms in the trap.

similar quality as in the repulsive case. However, for $N$ $\geqslant 1000$ the agreement quickly deteriorates, indicating that the whole mean field approximation breaks down. Indeed even quantally the solution of the GPE, Eq. (1) becomes unstable for $N>1500$ for $C s$ atoms.

In Figs. 5-8 we also show the densities (normalized to unity) and self-consistent potentials for the particle numbers $N=250$ and $N=1500$. We see that, whereas the case $N$ $=250$ is not dissimilar to the corresponding one with $a>0$, for $N=1500$ the situation becomes quite unfavorable for the TF approximation. This is for instance manifest in looking at the graph for the densities. In the attractive case TF and quantal solutions diverge with increasing $N$ whereas in the repulsive case they converge.

In Figs. 9 and 10 we plot the kinetic energy density per particle $(\tau / N)$ calculated quantally and in the TF approximation for $N=200000$ in the repulsive case and for $N=250$ in the attractive case. In these figures, the quantal kinetic energy density is given by

$$
\tau=|\nabla \psi|^{2}-\frac{1}{4} \Delta \rho=\frac{1}{4}\left[\frac{(\nabla \rho)^{2}}{\rho}-\Delta \rho\right]
$$

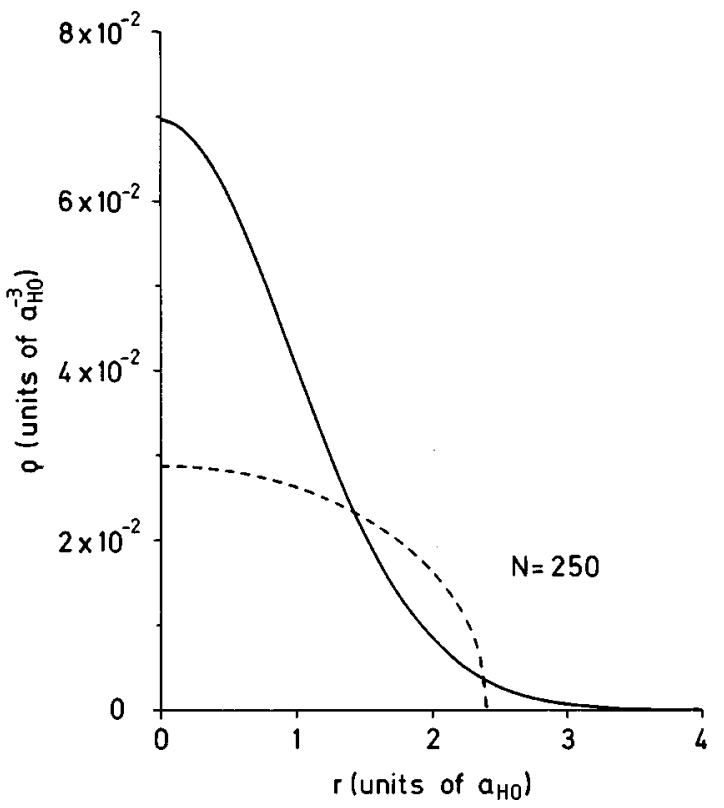

FIG. 5. The same as Fig. 1 but with 250 atoms in the trap in the attractive case.

in order to compare with the TF one according to Ref. [7].

For large number of particles, when the density profile has a relatively flat region at the interior (see Fig. 2), the quantal kinetic energy density is peaked at the surface [see Eq. (40)] whereas the TF one is rather a bulk term [see Eq. (31)]. Inspite of the rather different form of the quantal and $\mathrm{TF}$ kinetic energy densities in this case, the corresponding integrals are in good agreement (see Table II). This fact points again to the distribution character of the TF-kinetic energy density. However, if the number of particles is small, the quantal and TF kinetic energy density profiles are quite similar. This is due to the fact that the particle density fall off abruptly from $R=0$ (see Fig. 5) and consequently its derivatives contribute in all the range of $R$.

Other quantities where one directly needs the kinetic energy are, e.g., the collective monopole and quadrupole excitations of the condensate. Using the sum rule approach [16] they are given by: $\omega_{M}=\omega_{0}\left(5-e_{k i n} / e_{H O}\right)^{1 / 2}$ and $\omega_{D}$ $=\sqrt{2} \omega_{0}\left(1+e_{k i n} / e_{H O}\right)^{1 / 2}$. Table IV collects the results obtained for $\omega_{M}$ and $\omega_{D}$ calculated once quantally using the values obtained for $e_{k i n}$ and $e_{H O}$ from the solution of the GP

TABLE III. The same as in Table I but with a scattering length $\mathrm{a}=-1.0 \times 10^{-9} \mathrm{~m}$. The frequencies corresponding to the monopole $\left(\omega_{M}\right)$ and quadrupole $\left(\omega_{D}\right)$ calculated in the sum rule approach are also given.

\begin{tabular}{lcccccccc}
\hline \hline & QM & TF & QM & TF & QM & TF & QM & TF \\
\hline$N$ & 250 & 250 & 500 & 500 & 1000 & 1000 & 1500 & 1500 \\
$\mu$ & 1.424 & 1.437 & 1.338 & 1.369 & 1.120 & 1.212 & 0.691 & 1.009 \\
$e_{k i n}$ & 0.788 & 0.774 & 0.815 & 0.802 & 0.926 & 0.872 & 1.240 & 0.974 \\
$e_{H O}$ & 0.723 & 0.726 & 0.691 & 0.702 & 0.613 & 0.646 & 0.472 & 0.579 \\
$e_{\rho}$ & -0.039 & -0.032 & -0.084 & -0.070 & -0.209 & -0.153 & -0.511 & -0.272 \\
$\omega_{M}$ & 1.977 & 1.983 & 1.955 & 1.964 & 1.868 & 1.911 & 1.540 & 1.821 \\
$\omega_{D}$ & 2.044 & 2.033 & 2.088 & 2.070 & 2.240 & 2.168 & 2.693 & 2.316 \\
\hline \hline
\end{tabular}




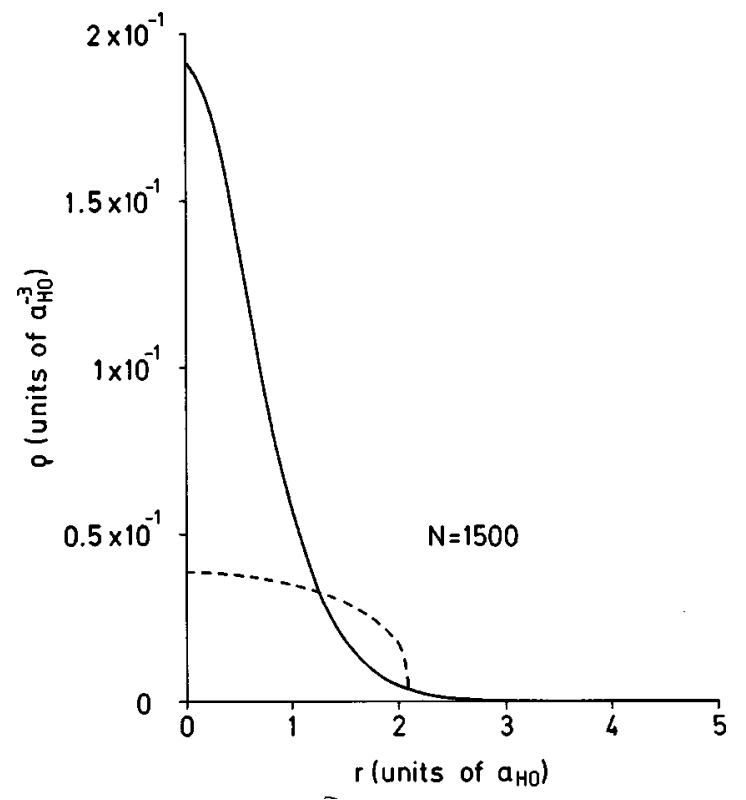

FIG. 6. The same as Fig. 1 but with 1500 atoms in the trap in the attractive case.

equation and also from the TF approach. The quantal results obtained by solving the time-dependent Gross-Pitaevskii equation [17] are also given for comparison. From this table one can see that the quantal results are very well reproduced for any number of atoms in the trap by the TF approximation presented in this paper. Table III also collects the quantal and TF frequencies for the monopole and quadrupole excitations obtained in the sum rule approach for the attractive case. From these results it can be seen that the TF values reproduce well the quantal ones in the region where the semiclassical approximation can be confidently applied $(N<1000)$.

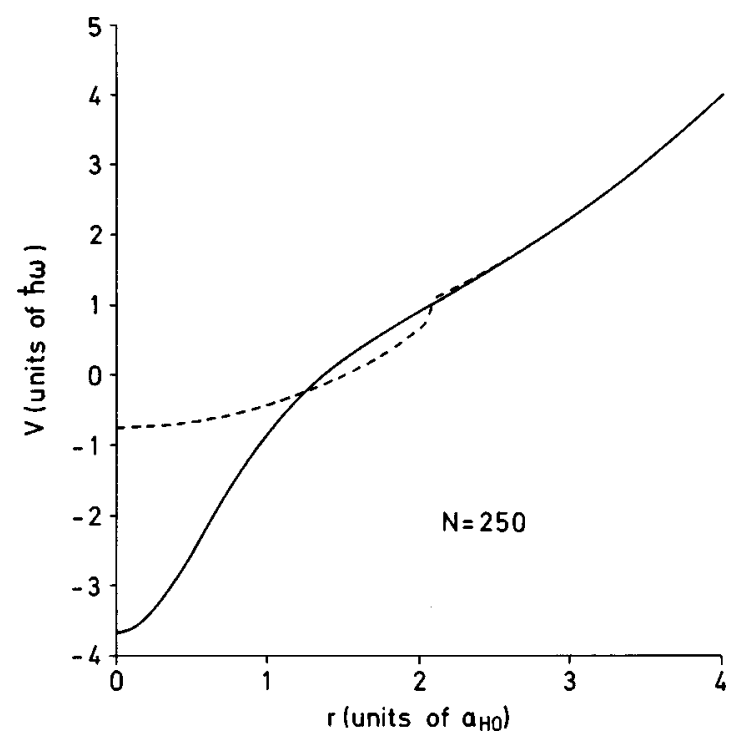

FIG. 7. The same as Fig. 3 but with 250 atoms in the trap in the attractive case.

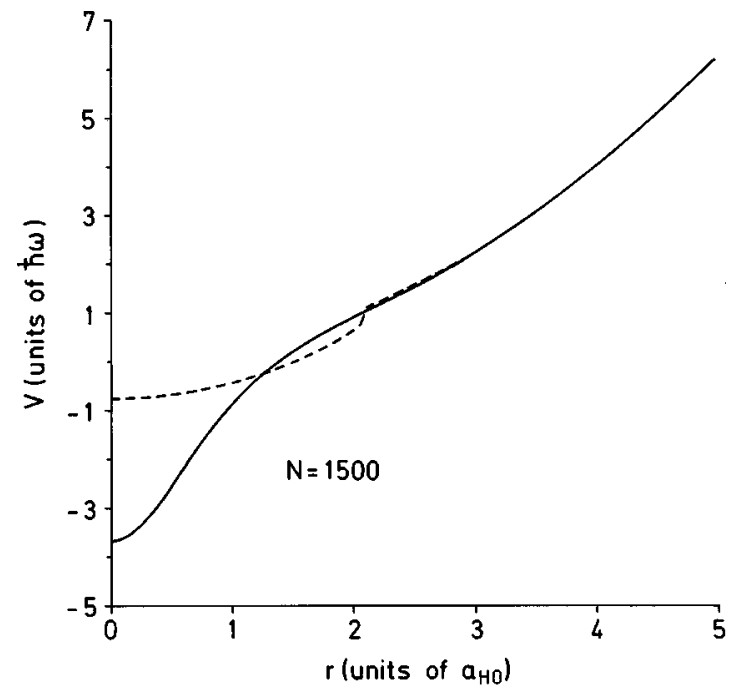

FIG. 8. The same as Fig. 3 but with 1500 atoms in the trap in the attractive case.

\section{DISCUSSION AND OUTLOOK}

In the preceding sections we have derived the ThomasFermi approximation, i.e., the $\hbar \rightarrow 0$ limit of the density matrix corresponding to the wave function of the GPE for the Bose condensate of atoms confined by magnetic traps. We have pointed out some misconceptions on this point that appeared in the past in the literature which for instance prevented the direct calculation of the kinetic energy in the large $N$ limit. On the contrary with our Thomas-Fermi approach the evaluation of the kinetic energy causes no problem and the results are globally in quite satisfactory agreement with the quantal solution of the Gross-Pitaevskii equation. For particle numbers where the kinetic energy represents a sig-

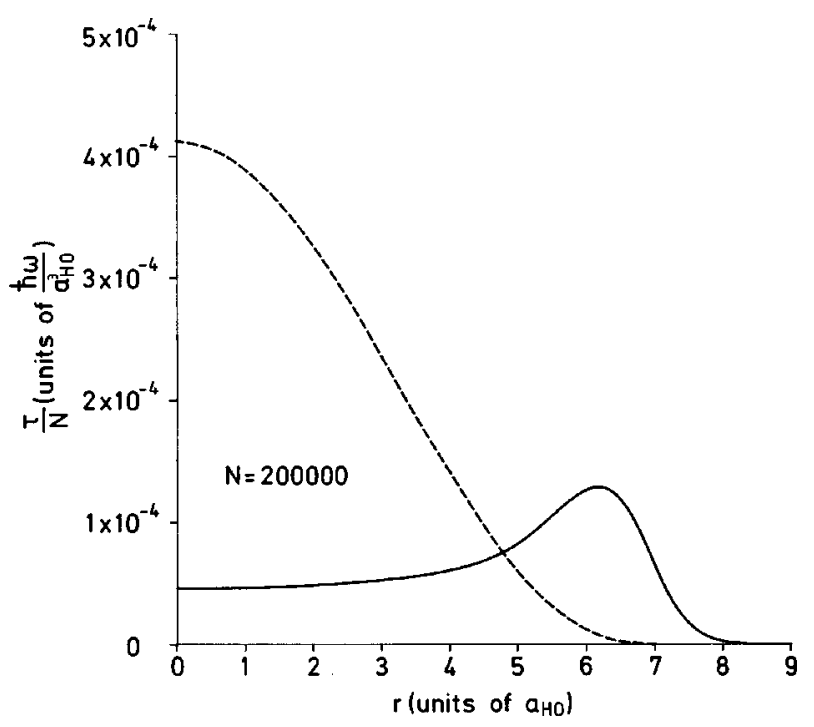

FIG. 9. Kinetic energy density per particle of a spherical trap (in $\hbar \omega a_{H O}^{-3}$ units) containing 200000 atoms as a function of the distance (in $a_{H O}$ units) in the repulsive case calculated from the solution of the GPE (solid line) and using the TF approach described in the text (dashed line). 


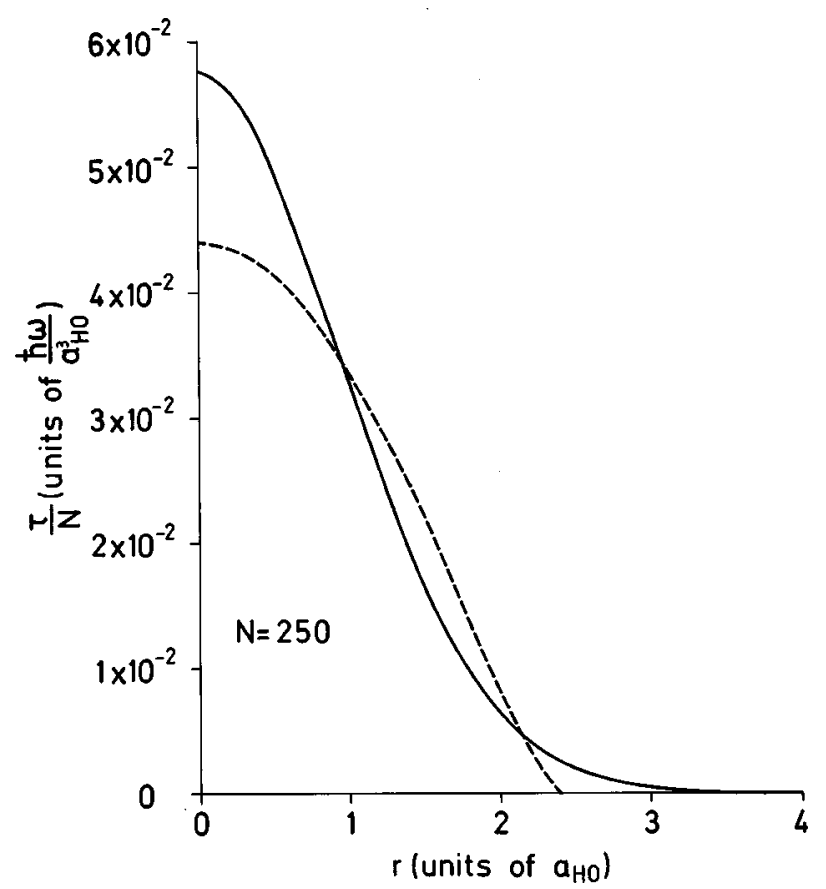

FIG. 10. The same as Fig. 9 but with 250 atoms in the trap in the attractive case.

nificant fraction of the total energy the $(\hbar=0) \mathrm{TF}$ expression yields very satisfying results as compared with the corresponding quantal values. As it has been discussed previously, for large $N$ values, nonanalytical quantal effects dominate the (small) kinetic energy and consequently this quantity cannot be well reproduced in a pure TF approximation in the large $N$ regime. The semiclassical theory of condensed inhomogeneous Bose gases has also been considered in some detail in Ref. [8]. There, however, the main emphasis was laid on the scattering terms out of the condensated (the Bogoliuvov approach). On the other hand no attempt was made to improve the kinetic energy in the pure $\hbar \rightarrow 0$ limit, i.e., in Thomas-Fermi approximation. However some estimates of the kinetic energy coming from gradients, i.e., $\hbar$ corrections are given in the large $N$ limit. On the contrary in the present work no quantal corrections to the lowest order Thomas-Fermi result have been worked out. Finally, we want to make some comments why for very large $N$ the ( $\hbar$ $=0) \mathrm{TF}$ values for the kinetic energy are very different (smaller) than the corresponding quantal values (see Table III). This simply means that the $\hbar=0$ part of the kinetic energy goes to zero much faster for large $N$ than for the full quantal solution. In other words, for very large $N$ the kinetic energy is dominated by the quantal corrections. Of course, finally for $N \rightarrow \infty$, the latter ones also drop to zero.

The above discussion shows again that the semiclassical approximations are a powerful tool but not devoid of subtleties and pitfalls. As a matter of fact also in this paper we, for simplicity, avoided developing the full complexity of the theory. One major simplification resides in the fact that we assume a spherical trap. This results in an isotropic momentum distribution $f_{0} \propto \delta\left(\mu-H_{c}\right)$ where $H_{c}=p^{2} / 2 m+V$ is the classical Hamiltonian. Deforming the trap leads to a non-
TABLE IV. Monopole $(L=0)$ and quadrupole $(L=2)$ frequencies $\omega$ calculated from the sum rule estimate using the GrossPitaevskii and Thomas-Fermi results for the kinetic energy. The results obtained by solving the time-dependent Gross-Pitaevskii equation are also given (TDGP label).

\begin{tabular}{ccccc}
\hline \hline$L$ & $N$ & $\omega(\mathrm{QM})$ & $\omega(\mathrm{TF})$ & $\omega(\mathrm{TDGP})$ \\
\hline 0 & \multirow{2}{*}{1000} & 2.095 & 2.098 & 2.114 \\
2 & & 1.794 & 1.788 & 1.734 \\
0 & \multirow{2}{*}{2000} & 2.137 & 2.137 & 2.145 \\
2 & & 1.694 & 1.701 & 1.650 \\
0 & \multirow{2}{*}{4000} & 2.166 & 2.166 & 2.172 \\
2 & & 1.617 & 1.617 & 1.579 \\
0 & \multirow{2}{*}{6000} & 2.180 & 2.180 & 2.185 \\
2 & & 1.579 & 1.579 & 1.545 \\
0 & \multirow{2}{*}{8000} & 2.189 & 2.191 & 2.192 \\
2 & & 1.556 & 1.549 & 1.525 \\
0 & \multirow{2}{*}{10000} & 2.194 & 2.197 & 2.198 \\
2 & & 1.539 & 1.531 & 1.511 \\
0 & \multirow{2}{*}{12000} & 2.199 & 2.202 & 2.202 \\
2 & & 1.526 & 1.517 & 1.500 \\
0 & \multirow{2}{*}{14000} & 2.202 & 2.206 & 2.205 \\
2 & & 1.517 & 1.507 & 1.493 \\
0 & \multirow{2}{*}{16000} & 2.205 & 2.208 & 2.207 \\
2 & & 1.509 & 1.499 & 1.486 \\
0 & 18000 & 2.207 & 2.211 & 2.209 \\
2 & & 1.503 & 1.492 & 1.481 \\
0 & 20000 & 2.209 & 2.213 & 2.211 \\
2 & & 1.497 & 1.486 & 1.477 \\
\hline \hline
\end{tabular}

trivial modification of the theory, since squeezing the condensate wave function in one direction and relaxing it in the other entails in turn a deformation of the momentum distribution which is opposite to the spatial one, i.e., momenta are strongest in the squeezed direction and lowest in the long direction of the deformation [15]. Our TF approach has also been useful for the evaluation of collective excitations of droplets of small or intermediate sizes. For this purpose, we have used the sum rule approach where the kinetic energy enters and for which we have used the TF values. In the deformed case the momentum becomes anisotropic. This has also been determined experimentally. The detailed determination of the anisotropy of the momenta (which may be position dependent) is theoretically not a completely trivial task in the general case and we will elaborate on this in future work. In the present case, however, there exists an evident first guess of the momentum deformation that results from a scaling argument of the harmonic oscillator coordinates. Assuming a prolate quadrupole deformation in the $z$ direction we have to replace the classical Hamiltonian in Eq. (10) by

$$
\widetilde{H}_{c}=\frac{1}{2 m}\left[\frac{\omega_{0}^{2}}{\omega_{\perp}^{2}}\left(p_{x}^{2}+p_{y}^{2}\right)+\frac{\omega_{0}^{2}}{\omega_{z}^{2}} p_{z}^{2}\right]+V\left(\frac{\omega_{\perp}}{\omega_{0}} x, \frac{\omega_{\perp}}{\omega_{0}} y, \frac{\omega_{z}}{\omega_{0}} z\right),
$$

where the ratios $\omega_{z} / \omega_{0}$ and $\omega_{\perp} / \omega_{0}$ are the frequency rela- 
tions in $z$ and $x, y$ with respect to the spherical case $\left(\omega_{0}\right)$. From Eq. (41) one easily calculates the so-called aspect ratio in the TF-approximation

$$
\sqrt{\frac{p_{z}^{2}}{p_{x}^{2}}}=\frac{\omega_{\perp}}{\omega_{z}}
$$

a result that has been given previously [3]. One other important consequence of the momentum deformation is that with Eq. (41) the moment of inertia of the condensate becomes equal to the irrotational flow value [16]. On the contrary using Eq. (10) with the isotropic momentum distribution the rigid momentum of inertia results. Consequently, the deformed case needs more detailed studies, which we reserve for future work. It is also evident that the present TF approach can be extended to finite temperature and to the Bogoliubov theory.
Another interesting subject of a more formal aspect is the evaluation of the $\hbar$-correction to the present lowest order theory. In principle this can easily be performed in posing in Eq. (3) $\rho=\rho_{0}+\hbar^{2} \rho_{2}$ and $\mu=\mu_{0}+\hbar^{2} \mu_{2}$ and properly sorting out different powers in $\hbar$. However, the proper elimination of divergencies and handling the normalization (19) and quantization (25) are slightly subtle problems. Investigations on the above mentioned directions are in progress.

\section{ACKNOWLEDGMENTS}

We want to thank S. Stringari for very useful discussions, L. P. Pitaevskii for his interest in this work, and M. Guilleumas for supplying us with the TDGP results. One of us (X.V.) also acknowledges financial support from DGCYT (Spain) under Grant No. PB95-1249 and from the DGR (Catalonia) under Grant No. 1998SGR-00011.
[1] L.P. Pitaevskii, Zh. Éksp. Teor. Phys. 40, 646 (1961) [Sov. Phys. JETP 13, 451 (1972)]; E.P. Gross, Nuovo Cimento 20, 454 (1961); J. Math. Phys. 4, 195 (1963).

[2] F. Dalfovo and S. Stringari, Phys. Rev. A 53, 2477 (1996).

[3] F. Dalfovo, L.P. Pitaevskii, and S. Stringari, J. Res. Natl. Inst. Stand. Technol. 101, 537 (1996).

[4] F. Dalfovo, S. Giorgini, L.P. Pitaevskii, and S. Stringari, e-print cond-mat/9806038.

[5] A.S. Parkins and D.F. Walls, Phys. Rep. 303, 1 (1998).

[6] L.H. Thomas, Proc. Cambridge Philos. Soc. 23, 542 (1926); E. Fermi, Z. Phys. 48, 73 (1928).

[7] P. Ring and P. Schuck, The Nuclear Many-Body Problem (Springer-Verlag, New York, 1980).

[8] E. Timmermans, P. Tommasini, and K. Huang, Phys. Rev. A 55, 3645 (1997).
[9] M.C. Gutzwiller, Chaos in Classical and Quantum Mechanics, Interdisciplinary Applied Mathematics (Springer-Verlag, New York, 1990).

[10] H. Krivine, M. Casas, and J. Martorell, Ann. Phys. (N.Y.) 200, 304 (1990).

[11] J. Carbonell, S. Shlomo, and P. Schuck (unpublished).

[12] A. Messiah, Quantum Mechanics (North-Holland, Amsterdam, 1961).

[13] C.C. Bradley, C.A. Sackett, J.J. Tollet, and R.G. Hullet, Phys. Rev. Lett. 75, 1687 (1995).

[14] M. Edwards and K. Burnett, Phys. Rev. A 51, 1382 (1995).

[15] B.K. Jennings, Phys. Lett. B 96, 1 (1980).

[16] S. Stringari, Phys. Rev. Lett. 77, 2360 (1996).

[17] M. Guilleumas (private communication). 